\title{
STATIC ANALYSIS OF CIRCULAR AND ELLIPTIC PLATES RESTING ON INTERNAL FLEXIBLE SUPPORTS BY THE BOUNDARY ELEMENT METHOD
}

\author{
Michat Guminiak, Krzysztof Szajek \\ Institute of Structural Engineering, Poznan University of Technology \\ Poznan, Poland \\ michal.guminiak@put.poznan.pl,krzysztof.szajek@put.poznan.pl
}

\begin{abstract}
A static analysis of circular and elliptic Kirchhoff plates resting on internal elastic supports by the Boundary Element Method is presented in the paper. Elastic support has the character of Winkler-type elastic foundations. Bilateral and unilateral internal constraints are taken into consideration. The Betti's theorem is used to derive the boundarydomain integral equation. The direct version of the boundary element method is presented and simplified boundary conditions, including curvilinear boundary elements, are introduced. The collocation version of boundary element method with non-singular approach is presented.
\end{abstract}

Keywords: internally supported plates, Boundary Element Method, fundamental solutions, curvilinear elements

\section{Introduction}

Plates resting on internal flexible constraints are often used in building structures. The analysis of internally supported plates in terms of the Boundary Element Method (BEM) has been the subject of numerous studies, e.g. [1-4]. The governing equation was formulated and derived using the direct approach. The BEM is the alternative way to the most popular Finite Element Method [5]. The BEM is often used in the theory of plates and is particularly suitable to analyse the plates of arbitrary shapes. The main advantage of BEM is its relative simplicity of formulating and solving problems of the potential theory and the theory of elasticity. Burczyński [6] described the BEM in a comprehensive manner and its application in a variety of fields, the theory of elasticity together with the appropriate solutions and a discussion of the basic types of boundary elements. Similarly, Wrobel and Aliabadi [7] presented applications of BEM in a wide range. Consideration of internal constraints requires modification of the boundary integral equations. According to the Bèzine approach [2], additional internal collocation points are introduced in which the forces or displacements are treated as unknown variables. This entails transformation of the pure boundary integral equation to boundary- 
domain integral equation. An alternative coupled BEM-flexibility force method was proposed by Rashed [4]. The major drawback of this approach is the necessity of the support to the edge of the plate providing geometric invariability of structure. In order to simplify the calculation procedures Guminiak et al. [8] proposed an alternative formulation of the boundary-domain integral equation for a thin plate. The authors used the Bèzine technique to establish deflections and forces of support reaction in internal collocation points. Katsikadelis et al. [9] used direct BEM approach to solve static and dynamic problem of plates with support condition inside a domain. Pawlak and Guminiak [10] applied the BEM and the FSM to solve similar problems considering unilateral internal constraints. Katsikadelis [11] described an application of BEM in a wide aspects of engineering analysis of the plates. The author also applied the Analog Equation Method (AEM) formulation in terms of BEM. The AEM approach also was used by Guminiak and Litewka [12] for rectangular thin plates resting on Winkler-type elastic foundation.

The present paper is devoted to application of BEM considering simplified boundary conditions for bending analysis of thin circular and elliptic plates resting on internal flexible support. In this approach there is no need to introduce the equivalent shear forces at the boundary and concentrated forces at the plate corners. Internal elastic support was introduced using the Bèzine technique.

\section{Integral formulation of thin plate bending}

A static problem of a plate resting on an internal flexible support is considered. Internal support has a discrete character. On the plate boundary, the following variables are considered: shear force $\widetilde{T}_{n}$, bending moment $M_{n}$ and deflection $w$, angle of rotation in normal direction $\varphi_{n}$ and angle of rotation in tangent direction $\varphi_{s}$. The expression $\widetilde{T}_{n}(\mathbf{y})=T_{n}(\mathbf{y})+R_{n}(\mathbf{y})$ denotes shear force for clamped and simplysupported edges

$$
\widetilde{T}_{n}(\mathbf{y})= \begin{cases}V_{n}(\mathbf{y}) & \text { on the boundary far from the corner } \\
R_{n}(\mathbf{y}) & \begin{array}{l}
\text { on a small fragment of the boundary } \\
\text { close to the corner }
\end{array}\end{cases}
$$

Because the relation between $\varphi_{s}(\mathbf{y})$ and the deflection is known: $\varphi_{s}(\mathbf{y})=d w(\mathbf{y}) / d s$ can be evaluated using a finite difference scheme of the deflection with two or more adjacent nodal values. In this analysis, the employed finite difference scheme includes the deflections of two adjacent nodes. The boundary-domain integral equations are derived using the Betti's theorem. Two plates are considered: the infinite plate, subjected unit concentrated loading and the real one. As a result, the first boundary-domain integral equation is in the form: 


$$
\begin{aligned}
& c(\mathbf{x}) \cdot w(\mathbf{x})+\int_{\Gamma}\left[T_{n}^{*}(\mathbf{y}, \mathbf{x}) \cdot w(\mathbf{y})-M_{n s}^{*}(\mathbf{y}, \mathbf{x}) \cdot \frac{d w(\mathbf{y})}{d s}-M_{n}^{*}(\mathbf{y}, \mathbf{x}) \cdot \varphi_{n}(\mathbf{y})\right] \cdot d \Gamma(\mathbf{y})= \\
& =\int_{\Gamma}\left[\widetilde{T}_{n}(\mathbf{y}) \cdot w^{*}(\mathbf{y}, \mathbf{x})-M_{n}(\mathbf{y}) \cdot \varphi_{n}^{*}(\mathbf{y}, \mathbf{x})\right] \cdot d \Gamma(\mathbf{y})+\int_{\Omega} p(\mathbf{y}) \cdot w^{*}(\mathbf{y}, \mathbf{x}) \cdot d \Omega(\mathbf{y})-\sum_{n=1}^{N} S_{n} \cdot w^{*}(n, \mathbf{x})
\end{aligned}
$$

where the fundamental solution of biharmonic equation $\nabla^{4} w=(1 / D) \bar{\delta}(\mathbf{y}-\mathbf{x})$ is given as a Green function

$$
w^{*}(\mathbf{y}, \mathbf{x})=\frac{1}{8 \pi D} r^{2} \ln r
$$

for a thin isotropic plate, $S_{n}$ expresses internal support reaction specified in internal collocation point, $r=|\mathbf{y}-\mathbf{x}|, \bar{\delta}$ is Dirac delta and $D=\left(E h_{\mathrm{p}}^{3}\right) /\left(12\left(1-v_{\mathrm{p}}^{2}\right)\right)$ is a plate stiffness. The coefficient $c(\mathbf{x})$ depends on the localization of point $\mathbf{x}$ and $c(\mathbf{x})=1$, when $\mathbf{x}$ is located inside the plate region, $c(\mathbf{x})=0.5$, when $\mathbf{x}$ is located on the smooth boundary and $c(\mathbf{x})=0$, when $\mathbf{x}$ is located outside the plate region.

The second boundary integral equation can be derived by substituting a unit concentrated force $P^{*}=1$ by unit concentrated moment $M_{n}^{*}=1$. It is equivalent to differentiate the first boundary-domain integral equation (2) on $n$ direction in point $\mathbf{x}$ on a plate boundary.

$$
\begin{aligned}
& c(\mathbf{x}) \cdot \varphi_{n}(\mathbf{x})+\int_{\Gamma}\left[\bar{T}_{n}^{*}(\mathbf{y}, \mathbf{x}) \cdot w(\mathbf{y})-\bar{M}_{n s}^{*}(\mathbf{y}, \mathbf{x}) \cdot \frac{d w(\mathbf{y})}{d s}-\bar{M}_{n}^{*}(\mathbf{y}, \mathbf{x}) \cdot \varphi_{n}(\mathbf{y})\right] \cdot d \Gamma(\mathbf{y})= \\
& =\int_{\Gamma}\left[\widetilde{T}_{n}(\mathbf{y}) \cdot \bar{w}^{*}(\mathbf{y}, \mathbf{x})-M_{n}(\mathbf{y}) \cdot \bar{\varphi}_{n}^{*}(\mathbf{y}, \mathbf{x})\right] \cdot d \Gamma(\mathbf{y})+\int_{\Omega} p(\mathbf{y}) \cdot \bar{w}^{*}(\mathbf{y}, \mathbf{x}) \cdot d \Omega(\mathbf{y})-\sum_{n=1}^{N} S_{n} \cdot \bar{w}^{*}(n, \mathbf{x})
\end{aligned}
$$

where

$$
\begin{aligned}
& \left\{\bar{T}_{n}^{*}(\mathbf{y}, \mathbf{x}), \bar{M}_{n}^{*}(\mathbf{y}, \mathbf{x}), \bar{M}_{n s}^{*}(\mathbf{y}, \mathbf{x}), \bar{w}^{*}(\mathbf{y}, \mathbf{x}), \bar{\varphi}_{n}^{*}(\mathbf{y}, \mathbf{x}), \bar{\varphi}_{s}^{*}(\mathbf{y}, \mathbf{x})\right\}= \\
& =\frac{\partial}{\partial n(\mathbf{x})}\left\{T_{n}^{*}(\mathbf{y}, \mathbf{x}), M_{n}^{*}(\mathbf{y}, \mathbf{x}), M_{n s}^{*}(\mathbf{y}, \mathbf{x}), w^{*}(\mathbf{y}, \mathbf{x}), \varphi_{n}^{*}(\mathbf{y}, \mathbf{x}), \varphi_{s}^{*}(\mathbf{y}, \mathbf{x})\right\}
\end{aligned}
$$

\section{Types of boundary elements}

In the simplest approach, the boundary element of the constant type is introduced (Fig. 1a). It is also possible to define the geometry of the element considering three nodal points and only one collocation point connected with the relevant physical boundary value (Fig. 1b). The collocation point may be located slightly 
outside of a plate edge. The geometry of the element can be defined using polynominal function, described in standard coordinate system $\langle-1,0,1\rangle$. These functions are in the form:

$$
N_{1}=\frac{1}{2} \eta \cdot(\eta-1), N_{2}=1-\eta^{2}, N_{3}=-\frac{1}{2} \eta \cdot(\eta-1)
$$

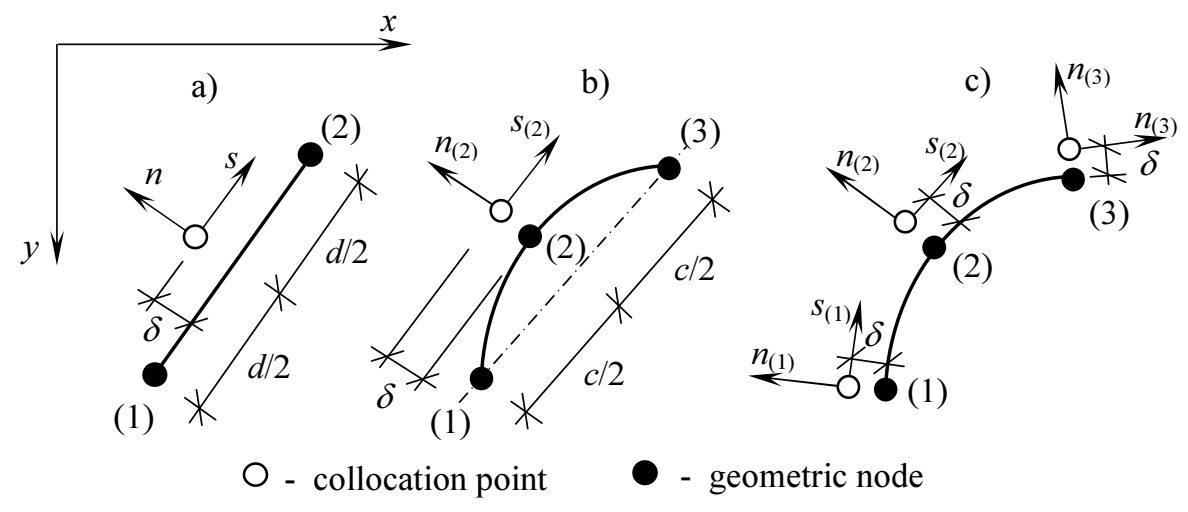

Fig. 1. Boundary elements of the constant type in non-singular approach

A quadratic curvilinear isoparametric element is shown in Figure 1c. According to the non-singular approach, the boundary (boundary-domain) integral equations can be formulated using the approach of single collocation point associated with each boundary element of the constant type and single collocation point associated with each geometric node of the quadratic element.

\section{Assembly of the set of algebraic equation}

Let it be assumed that a plate boundary is discretized using constant elements. Internal flexible support can be treated as the Winkler-type foundations, where the support reaction $S_{n}$ can be expressed in the simple form

$$
S_{n}=k_{n} \cdot w_{n}
$$

where $k_{n}$ and $w_{n}$ are the support stiffness and displacement. In the case of the free edge, the characteristic matrix must be expanded using additional components $\mathbf{G}_{\mathrm{BS}}$ and $\boldsymbol{\Delta}$ :

$$
\left[\begin{array}{ccc}
\mathbf{G}_{\mathrm{BB}} & \mathbf{G}_{\mathrm{BS}} & \mathbf{G}_{\mathrm{Bw}} \\
\Delta & -\mathbf{I} & \mathbf{0} \\
\mathbf{G}_{\mathbf{w B}} & \mathbf{G}_{\mathrm{wS}} & \mathbf{G}_{\mathrm{ww}}
\end{array}\right] \cdot\left\{\begin{array}{l}
\mathbf{B} \\
\varphi_{\mathrm{s}} \\
\mathbf{w}
\end{array}\right\}=\left\{\begin{array}{c}
F_{\mathrm{B}} \\
\mathbf{0} \\
\mathbf{F}_{\mathrm{w}}
\end{array}\right\}
$$


and $\mathbf{B}$ is the vector of boundary independent variables, $\varphi_{\mathbf{s}}$ is the vector of additional parameters of the angle of rotation in the tangential direction, which depend on the boundary deflection in case of the free edge, $\mathbf{G}_{\mathbf{B B}}$ is the matrix grouping boundary integrals dependent on type of boundary. Matrix $\mathbf{G}_{\mathbf{B S}}$ groups boundary integrals of functions $M_{n s}^{*}$ and $\bar{M}_{n s}^{*}$ in case of free edge occurence and it is the additional matrix grouping boundary integrals corresponding with rotation in tangential direction $\varphi_{\mathbf{s}}$. The matrix $\mathbf{G}_{\mathbf{B w}}$ groups values of fundamental functions $w^{*}$ and $\bar{w}^{*}$ established in internal collocation points associated with internal constraints. The matrix $\Delta$ groups the finite difference expressions for the angle of rotation in the tangential direction $\varphi_{\mathbf{s}}$ in terms of deflections at suitable, adjacent nodes and $\mathbf{I}$ is the unit matrix. In the computer program deflections at two neighbouring nodes are used. Hence, for a clamped edge, a simply-supported edge and a free edge, two independent unknowns are always considered. Matrices $\mathbf{G}_{\mathbf{w B}}, \mathbf{G}_{\mathbf{w S}}$ and $\mathbf{G}_{\mathbf{w w}}$ group boundary integrals and values of fundamental function $w^{*}$ calculates in collocation points associated with internal supports respectively. All of the designations are shown in Figure 2.

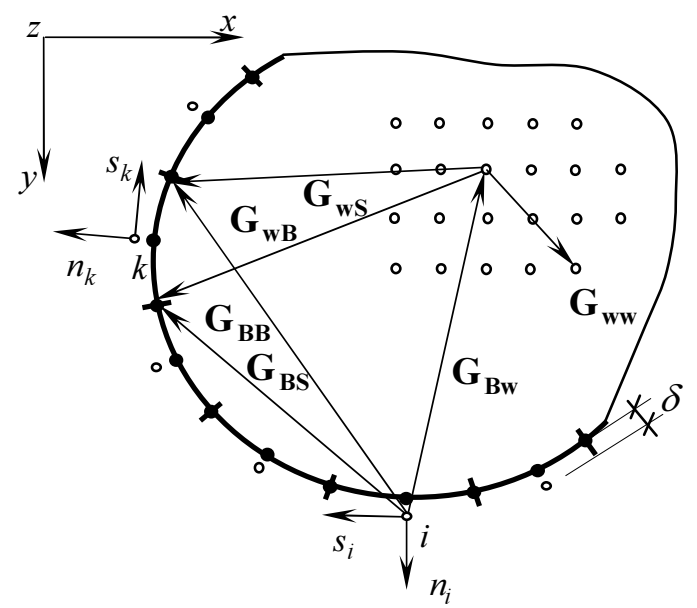

Fig. 2. Construction of characteristic matrix

\subsection{Construction of characteristic matrix}

The boundary-domain integral equation will be formulated in a non-singular approach. To construct the characteristic matrix $\mathbf{G}$, integration of suitable fundamental function on boundary is needed. Integration is done in a local coordinate system $n_{i}, s_{i}$ connected with $i^{\text {th }}$ boundary element and next, these integrals must be transformed to $n_{k}, s_{k}$ coordinate system, connected with $k^{\text {th }}$ element. Localization of collocation point is defined by the parameter $\delta$ or non-dimensional parameter $\varepsilon$. 
This parameter can be defined as $\varepsilon=\delta / d$ or $\varepsilon=\delta / c$ (Fig. 1). To calculate elements of the characteristic matrix there are applied the following methods: a) classic, numerical Gauss procedure for non-quasi diagonal elements or $b$ ) modified, numerical integration of Gauss method for quasi-diagonal elements proposed by Litewka and Sygulski [13]. The authors proposed inverse localization of the Gauss points in domain of integration, which is illustrated in Figure 3. Boundary integrals on curved element are calculated according to Gauss method. Integrals of fundamental functions over the plate edge are calculated using $n_{i}, s_{i}$ coordinate system, connected with $i^{\text {th }}$ physical node. Then, they are transformed to $n_{k}, s_{k}$ coordinate system [ 8 , $14,15]$.

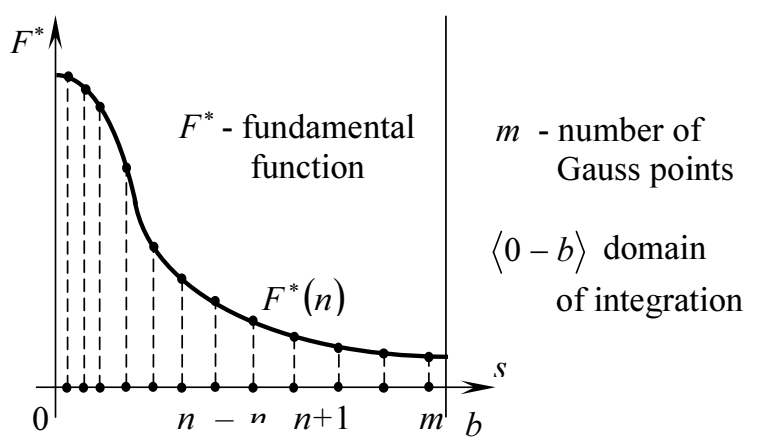

Fig. 3. Calculation of quasi-diagonal integrals using modified Gauss method [13]

In case of consideration of a free edge, the angle of rotation in a tangent direction can be expressed by deflection of two neighbouring nodes

$$
\varphi_{s}^{(i-1)}=\varphi_{s}^{(i)}=\varphi_{s}^{(i+1)}=\left(w_{b}^{(i+1)}-w_{b}^{(i)}\right) / d_{i+1}
$$

where $d_{i}$ is the projection of a section connecting physical nodes (collocation points) $i$ and $i+1$ on the line tangential to the boundary element in collocation point $i^{\text {th }}$. It is also assumed that a plate can be supported on boundary and also resting on unilateral internal supports. In the last case, especially when a plate is supported only inside its domain, the problem can be solved iteratively. On each iteration step the collocation points inside a plate with a negative value of support reaction are switched off. The process can be stopped, when between the iterations there is no change of sign of any reaction.

\subsection{Construction of right-hand-side vector}

It is assumed that constant loading $p$ is acting on a plate surface. Integrals $p \int_{\Omega} w^{*} d \Omega$ and $p \int_{\Omega} \bar{w}^{*} d \Omega$ can be evaluated analytically in terms of the Abdel-Akher and Hartley proposition (contour of loading is expressed in polygonal form) [16]. 


\section{Calculation of deflection, angle of rotation, bending and torsional moments inside a plate domain}

The solution of algebraic equations allows one to determine the boundary variables. Then, it is possible to calculate the deflection, angle of rotation in an arbitrary direction, bending and torsional moments at an arbitrary point of the plate domain. Each value can be expressed as the sum of three variables depending on the boundary variables $\overline{\mathbf{B}}$, external loading $p$ and reaction of internal supports $\mathbf{S}$, for example deflection

$$
w=w(\overline{\mathbf{B}})+w(p)+w(\mathbf{S})
$$

where $\overline{\mathbf{B}}=\left\{\mathbf{B} \varphi_{\mathbf{S}}\right\}^{T}$. A similar relation can be applied to establish the angle of rotation in an arbitrary direction. In terms of the thin plate theory, the bending moments and torsional moment are given in the classic form $M_{x}(x, y)=-D\left(w_{x x}+v w,_{y y}\right), M_{y}(x, y)=-D\left(w_{, y y}+v w,_{x x}\right)$ and $M_{x y}(x, y)=-D(1-v) \cdot w,_{x y}$ and $w(x, y)$ is the function of displacements and $x, y$ are the global coordinates of an arbitrary point.

\section{Numerical examples}

Circular and elliptic plates with various boundary conditions are considered. Plates are subjected only to a uniformly distributed loading $p=1.0 \mathrm{kN} / \mathrm{m}$ on the entire surface or concentrated force $P=10.0 \mathrm{kN}$ at its centre. Twenty Gauss points are applied to evaluate boundary integrals. Circular plates are divided by boundary elements with the same length. For elliptic plate localization of geometrical nodes on the edge for 32 boundary elements is presented in Figure 4. For 64 boundary elements, similar localization is assumed, dividing all of segments: $l, l / 2, l / 3$ and $l / 6$ by halves.

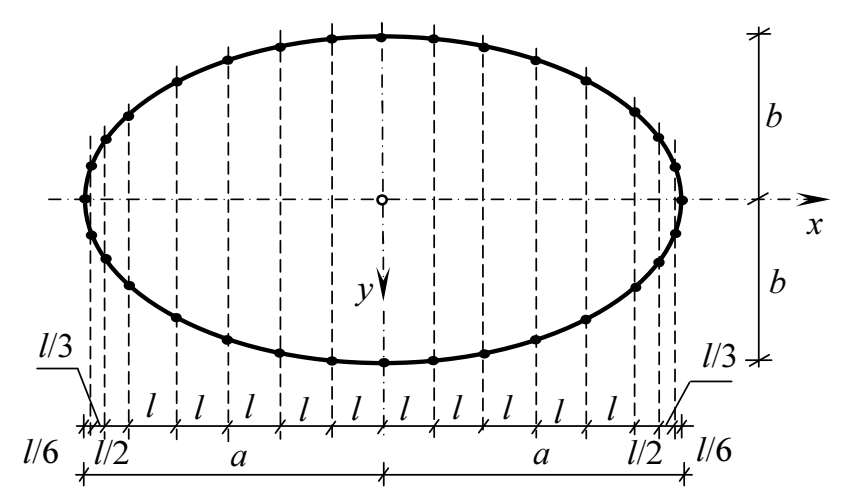

Fig. 4. Localization of boundary elements inscribed in ellipse contour 
Localization of internal collocation points corresponding to the number of 128 discrete supports localized inside a plate domain which for circular plates is shown in the Figure 5.

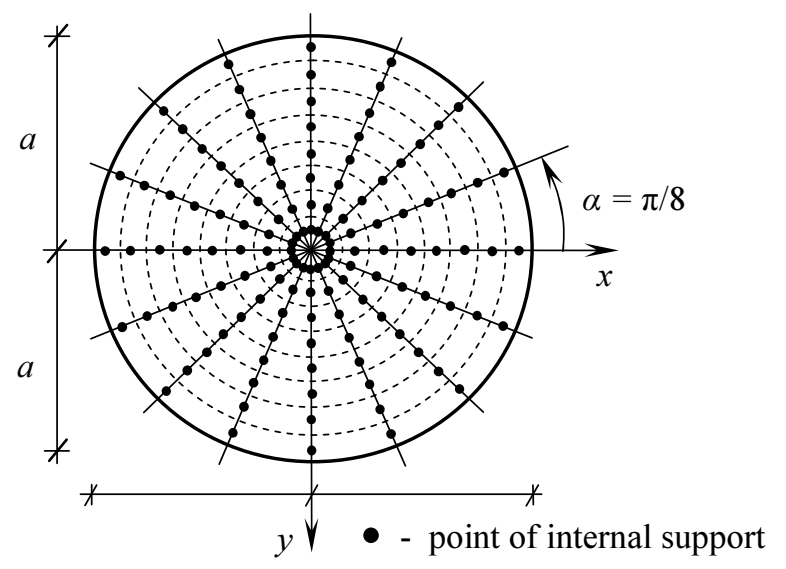

Fig. 5. Localization of internal collocation points for a circular plate

For an elliptic plate, coordinates of internal collocation point are assumed according to polar coordinates. The radius to the selected collocation point is expressed as follows:

$$
r=\sqrt{b^{2} /\left(1-e^{2} \cdot \cos ^{2} \alpha\right)}
$$

where $e^{2}=\left(a^{2}-b^{2}\right) / a^{2}$. In the considered examples, angle $\alpha$ is the multiple of $\pi / 8 \mathrm{rad}$. Localization of internal collocation points for number of 128 discrete supports is presented in Figure 6.

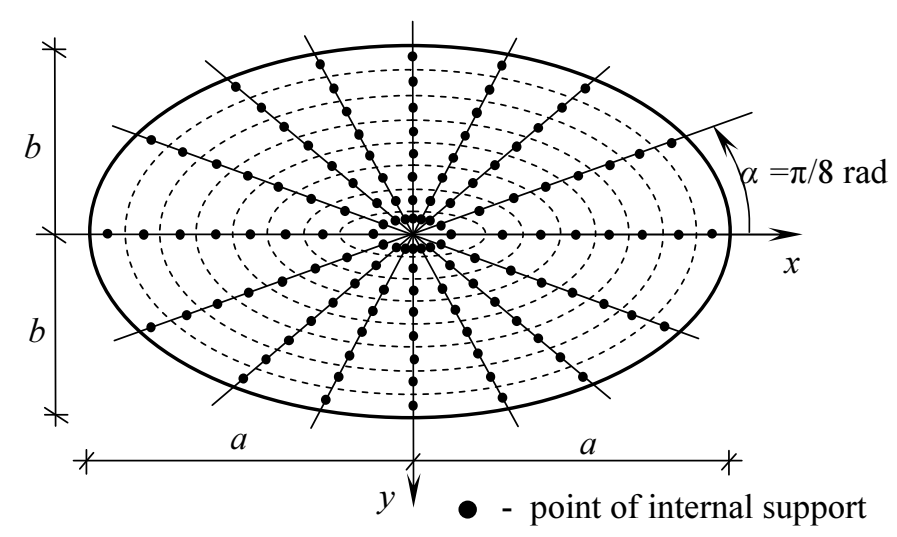

Fig. 6. Localization of internal collocation points for an elliptic plate 
The following plate properties are assumed: $E=205.0 \mathrm{GPa}$ and $v=0.3$, thickness $h=0.01 \mathrm{~m}$. Internal support stiffness $k_{i}=50.0 \mathrm{kN} / \mathrm{m}$. The circular plate radius is equal to $a=2.0 \mathrm{~m}$. Elliptic plate half-axes are equal to $a=3.0 \mathrm{~m}$ and $b=2.0 \mathrm{~m}$. A numerical analysis was conducted using following boundary and finite element discretization: BEM I - rectilinear boundary element of the constant type, $\varepsilon=\delta / d=0.01$; BEM II - curved, simplified boundary element of the constant type, $\varepsilon=\delta / c=0.1$; BEM III - three-node isoparametric curved boundary element, $\varepsilon=\delta / c=0.1 ;$ FEM - finite element analysis was carried out using Abaqus/STANDARD v6.12 computational program and eight-node doubly curved shell finite element with reduced integration (S8R) was adopted [17]. The plate domain was divided into 3936 and 4706 elements for a circular and elliptic plate, respectively. The results of calculation for circular plate clamped on a whole edge, resting on internal supports and subjected to the uniformly distributed loading $p$ are presented in Tables 1 and 2.

Table 1

Deflection at the plate centre

\begin{tabular}{|c|c|c|c|}
\hline \multirow{2}{*}{$\begin{array}{c}\text { Number of boundary } \\
\text { elements }\end{array}$} & \multicolumn{3}{|c|}{$\widetilde{w}=w D / p a^{4}$} \\
\cline { 2 - 3 } & BEM I & BEM III & FEM \\
\hline 32 & $9.8428 \cdot 10^{-4}$ & $9.8438 \cdot 10^{-4}$ & \multirow{2}{*}{$9.8558 \cdot 10^{-4}$} \\
\hline 64 & $9.8476 \cdot 10^{-4}$ & $9.8480 \cdot 10^{-4}$ & \\
\hline
\end{tabular}

Table 2

Bending moments

\begin{tabular}{|c|c|c|c|c|}
\hline \multirow{2}{*}{$\begin{array}{c}\text { Number of boundary } \\
\text { elements }\end{array}$} & \multicolumn{4}{|c|}{$\tilde{M}=M_{r} / p a^{2}$} \\
\cline { 2 - 5 } & \multicolumn{2}{|c|}{ At the centre } & \multicolumn{2}{c|}{ On boundary } \\
\cline { 2 - 5 } & BEM I & BEM III & BEM I & BEM III \\
\hline 32 & -0.021265 & -0.021290 & -0.040548 & -0.040468 \\
\hline 64 & -0.021424 & -0.021430 & -0.040333 & -0.040317 \\
\hline
\end{tabular}

Results of calculation for circular plate simply-supported on a whole edge, resting on internal supports and subjected to the uniformly distributed loading $p$ are presented in Tables 3 and 4 .

Deflection at the plate centre

\begin{tabular}{|c|c|c|c|}
\hline \multirow{2}{*}{$\begin{array}{c}\text { Number of boundary } \\
\text { elements }\end{array}$} & \multicolumn{3}{|c|}{$\widetilde{w}=w D / p a^{4}$} \\
\cline { 2 - 3 } & BEM I & BEM II & FEM \\
\hline 32 & $1.0148 \cdot 10^{-3}$ & $1.0150 \cdot 10^{-3}$ & \multirow{2}{*}{$1.0137 \cdot 10^{-3}$} \\
\hline 64 & $1.0136 \cdot 10^{-3}$ & $1.0137 \cdot 10^{-3}$ & \\
\hline
\end{tabular}


Table 4

Bending moment at the plate centre

\begin{tabular}{|c|c|c|}
\hline \multirow{2}{*}{$\begin{array}{c}\text { Number of boundary } \\
\text { elements }\end{array}$} & \multicolumn{2}{|c|}{$\tilde{M}=M_{r} / p a^{2}$} \\
\cline { 2 - 3 } & BEM I & BEM II \\
\hline 32 & -0.029675 & -0.029704 \\
\hline 64 & -0.029809 & -0.029822 \\
\hline
\end{tabular}

Results of calculation for circular plate with a whole edge free, resting on internal supports and subjected to the concentrated force $P$ at the centre are presented in Table 5 and Figure 7.

Table 5

Deflection at the plate centre

\begin{tabular}{|c|c|c|c|c|}
\hline \multirow{2}{*}{$\begin{array}{c}\text { Number of boundary } \\
\text { elements }\end{array}$} & \multicolumn{4}{|c|}{$\widetilde{w}=w D / P a^{2}$} \\
\cline { 2 - 5 } & \multicolumn{2}{|c|}{ Bilateral } & Unilateral & Bilateral \\
\cline { 2 - 4 } & BEM I & BEM II & BEM I & FEM \\
\hline 32 & $2.8556 \cdot 10^{-3}$ & $2.8557 \cdot 10^{-3}$ & $2.9135 \cdot 10^{-3}$ & \multirow{2}{*}{$2.8676 \cdot 10^{-3}$} \\
\hline 64 & $2.8558 \cdot 10^{-3}$ & $2.8558 \cdot 10^{-3}$ & $2.9146 \cdot 10^{-3}$ & \\
\hline
\end{tabular}

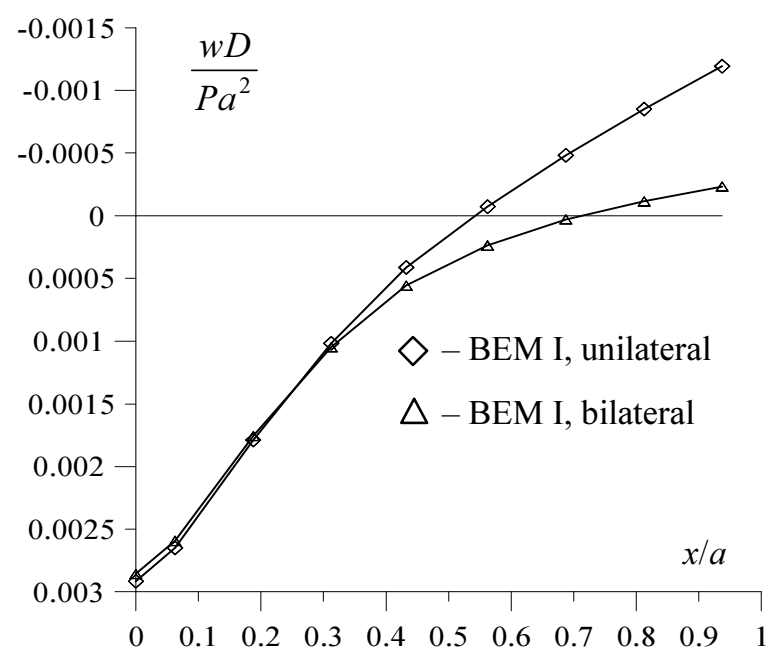

Fig. 7. Deflection along plate radius $r / a$

Results of calculation for an elliptic plate with a whole edge free, resting on internal supports and subjected to the concentrated force $P$ at the centre are presented in Table 6 and Figure 8. 
Table 6

Deflection at the plate centre

\begin{tabular}{|c|c|c|c|c|}
\hline \multirow{2}{*}{$\begin{array}{c}\text { Number of boundary } \\
\text { elements }\end{array}$} & \multicolumn{4}{|c|}{$\widetilde{w}=w D / P b^{2}$} \\
\cline { 2 - 4 } & \multicolumn{2}{|c|}{ Bilateral } & Unilateral & Bilateral \\
\cline { 2 - 4 } & BEM I & BEM II & BEM I & FEM \\
\hline 32 & $3.2067 \cdot 10^{-3}$ & $3.1256 \cdot 10^{-3}$ & $3.2773 \cdot 10^{-3}$ & \multirow{2}{*}{$3.1116 \cdot 10^{-3}$} \\
\hline 64 & $3.2067 \cdot 10^{-3}$ & $3.2266 \cdot 10^{-3}$ & $3.2773 \cdot 10^{-3}$ & \\
\hline
\end{tabular}

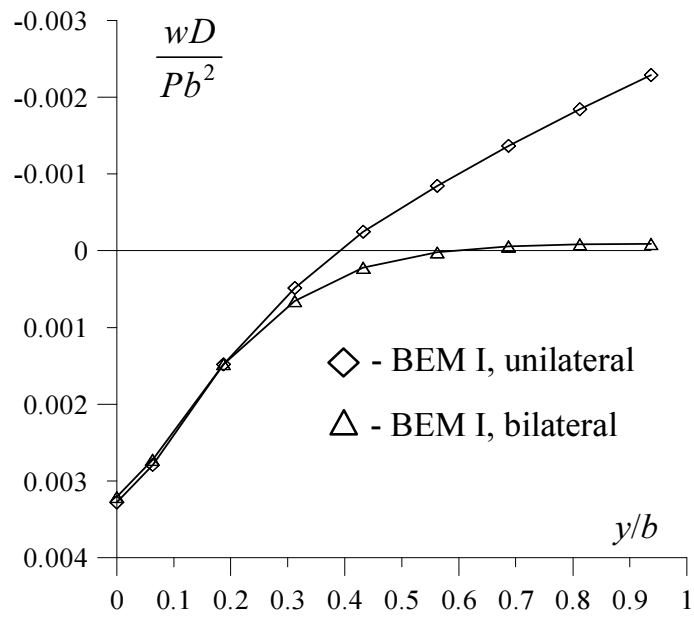

Fig. 8. Deflection along half-axis $x / b$

\section{Conclusions}

Static analysis of plates resting on internal elastic supports using the Boundary Element Method is presented. The problem was solved using the Kirchhoff theory of plates with the modified approach, in which the boundary conditions are defined so that there is no need to introduce equivalent boundary quantities dictated by the boundary value problem for the biharmonic differential equation even if typical rectilinear boundary element was used. The Bèzine technique and static fundamental solution for a usual thin plate were used to introduce supports inside a plate domain and establish their reactions and deflections. The modified Gauss method [13] was used to calculate quasi-diagonal integrals for curved boundary elements. The application of curved boundary elements gives a similar result as in the case of typical rectilinear elements. Direct collocation non-singular BEM formulation of plate bending considering internal supports in case of a large number of unknowns may lead to wrong conditioning of the characteristic matrix. The analysis of influence of collocation point localization on conditioning of characteristic matrix was carried out in [18]. 


\section{References}

[1] Bèzine G., A boundary integral equation method for plate flexure with condition inside the domain, International Journal of Numerical Method in Engineering 1981, 15, 1647-1657.

[2] $\mathrm{Bu}$ X.M., Yan Z.D., Bending problems of rectangular thin plate with free edges laid on tensionless Winkler foundation, Applied Mathematic and Mechanics 1989, 10, 5, 435-442.

[3] Xiao J.R., Boundary element analysis of unilateral supported Reissner plates on elastic foundations, Computational Mechanics 2001, 27, 1-10.

[4] Rashed Y.F., A coupled BEM-flexibility force method for bending analysis of internally supported plates, International Journal of Numerical Method in Engineering 2002, 54, 1431-457.

[5] Stern M., A general boundary integral formulation for the numerical solution of plate bending problems, International Journal of Solids and Structures 1978, 15, 769-782.

[6] Burczyński T., The Boundary Element Method in Mechanics, WNT, Warszawa 1995 (in Polish).

[7] Wrobel L.C., Aliabadi M.H., The Boundary Element Methods in Engineering, McGraw-Hill College, 2002.

[8] Guminiak M., Litewka P., Sygulski R., Static analysis of plates on the unilateral foundation by BEM, Proceedings of the $9^{\text {th }}$ International Conference Modern Building Materials, Structures and Techniques, Vilnius, May 19-21, 2004, Selected papers, (eds.) E.K. Zavadskas, P. Vainiunas, F.M. Mazzolani, Vilnius Gediminas Technical University Press "Technika" Scientific book 1026, 2004, vol. III, 765-768.

[9] Katsikadelis J.T., Sapountzakis E.J., Zorba E.G., A BEM approach to static and dynamic analysis with internal supports, Computational Mechanics 1990, 7, 1, 31-40.

[10] Pawlak Z., Guminiak M., The application of fundamental solutions in static analysis of thin plates resting on the internal elastic support, Foundations of Civil and Environmental Engineering 2008, 11, 67-96

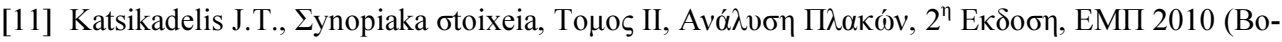
undary elements, Vol. II, Analysis of Plates, Second Edition, NTUA, Athens 2010).

[12] Guminiak M., Litewka B., Selected problems of thin and thick plates theory in terms of BEM, Foundations of Civil and Environmental Engineering 2012, 15, 41-90.

[13] Litewka B., Sygulski R., Application of the fundamental solutions by Ganowicz in a static analysis of Reissner's plates by the boundary element method, Engineering Analysis with Boundary Elements 2010, 34, 1072-1081.

[14] Guminiak M., Application of simplified curved boundary elements to the plate analysis - part one, Scientific Research of the Institute of Mathematics and Computer Science 2010, 2, 9 , 59-71.

[15] Guminiak M., Application of simplified curved boundary elements to the plate analysis - part two, Scientific Research of the Institute of Mathematics and Computer Science 2010, 2, 9, 73-81.

[16] Abdel-Akher A., Hartley G.A., Evaluation of boundary integrals for plate bending, International Journal of Numerical Method in Engineering 1989, 28, 75-93.

[17] Abaqus, Abaqus Manuals. Inc. Providence, 2005.

[18] Guminiak M., Static analysis of thin plates by the Boundary Element Method in a non-singular approach, Foundations of Civil and Environmental Engineering 2007, 9, 75-93. 